\title{
U-Statistic for Multivariate Stable Distributions
}

\author{
Mahdi Teimouri, Saeid Rezakhah, and Adel Mohammadpour
}

Department of Statistics, Faculty of Mathematics and Computer Science, Amirkabir University of Technology (Tehran Polytechnic), 424 Hafez Ave., Tehran 15914, Iran

Correspondence should be addressed to Adel Mohammadpour; adel@aut.ac.ir

Received 5 December 2016; Revised 9 February 2017; Accepted 19 February 2017; Published 3 April 2017

Academic Editor: Steve Su

Copyright (C) 2017 Mahdi Teimouri et al. This is an open access article distributed under the Creative Commons Attribution License, which permits unrestricted use, distribution, and reproduction in any medium, provided the original work is properly cited.

A $U$-statistic for the tail index of a multivariate stable random vector is given as an extension of the univariate case introduced by Fan (2006). Asymptotic normality and consistency of the proposed $U$-statistic for the tail index are proved theoretically. The proposed estimator is used to estimate the spectral measure. The performance of both introduced tail index and spectral measure estimators is compared with the known estimators by comprehensive simulations and real datasets.

\section{Introduction}

In recent years, stable distributions have received extensive use in a vast number of fields including physics, economics, finance, insurance, and telecommunications. Different sorts of data found in applications arise from heavy tailed or asymmetric distribution, where normal models are clearly inappropriate. In fact, stable distributions have theoretical underpinnings to accurately model a wide variety of processes. Stable distribution has originated with the work of Lévy [1]. There are a variety of ways to introduce a stable random vector. In the following, two definitions are proposed for a stable random vector; see Samorodnitsky and Taqqu [2].
Definition 1. A random vector $\mathbf{X}=\left(X_{1}, \ldots, X_{d}\right)^{T}$ is said to be stable in $\mathbb{R}^{d}$ if for any positive numbers $A$ and $B$ there are a positive number $C$ and a vector $\mathbf{D} \in \mathbb{R}^{d}$ such that

$$
A \mathbf{X}_{1}+B \mathbf{X}_{2} \stackrel{d}{=} C \mathbf{X}+\mathbf{D}
$$

where $\mathbf{X}_{1}$ and $\mathbf{X}_{2}$ are independent and identical copies of $\mathbf{X}$ and $C=\left(A^{\alpha}+B^{\alpha}\right)^{1 / \alpha}$.

Definition 2. Let $0<\alpha<2$. Then $\mathbf{X}$ is a non-Gaussian $\alpha$ stable random vector in $\mathbb{R}^{d}$ if there exist a finite measure $\Gamma$ on the unit sphere $\mathbb{S}^{d}=\left\{\mathbf{x}=\left(x_{1}, \ldots, x_{d}\right)^{T} \in \mathbb{R}^{d} \mid\langle\mathbf{x}, \mathbf{x}\rangle=1\right\}$ and a vector $\boldsymbol{\mu}=\left(\mu_{1}, \ldots, \mu_{d}\right)^{T} \in \mathbb{R}^{d}$ such that

$$
\varphi_{\mathbf{X}}(\mathbf{t})=\log E(\exp (i\langle\mathbf{t}, \mathbf{X}\rangle))= \begin{cases}-\int_{\mathbb{S}^{d}}|\langle\mathbf{t}, \mathbf{s}\rangle|^{\alpha}\left[1-i \operatorname{sgn}\langle\mathbf{t}, \mathbf{s}\rangle \tan \left(\frac{\pi \alpha}{2}\right)\right] \Gamma(d \mathbf{s})+i\langle\mathbf{t}, \boldsymbol{\mu}\rangle, & \alpha \neq 1, \\ -\int_{\mathbb{S}^{d}}|\langle\mathbf{t}, \mathbf{s}\rangle|\left[1+i \operatorname{sgn}\langle\mathbf{t}, \mathbf{s}\rangle \frac{2}{\pi} \log |\langle\mathbf{t}, \mathbf{s}\rangle|\right] \Gamma(d \mathbf{s})+i\langle\mathbf{t}, \boldsymbol{\mu}\rangle, & \alpha=1,\end{cases}
$$

where $\langle\mathbf{t}, \mathbf{s}\rangle=\sum_{i=1}^{d} t_{i} s_{i}$ for $\mathbf{t}=\left(t_{1}, \ldots, t_{d}\right)^{T}, \mathbf{s}=\left(s_{1}, \ldots, s_{d}\right)^{T}$, $i^{2}=-1$, and $\operatorname{sgn}(\cdot)$ denotes the sign function. The pair $(\Gamma, \boldsymbol{\mu})$ is unique.

The parameter $\alpha$, in Definitions 1 and 2 , is called tail index. A random vector $\mathbf{X}$ is said to be a strictly $\alpha$-stable random vector in $\mathbb{R}^{d}$ if $\boldsymbol{\mu}=\mathbf{0}$ for $\alpha \neq 1$; see Samorodnitsky and Taqqu [2]. We note that $\mathbf{X}$ is strictly $\alpha$-stable, in the sense of Definition 1, if $\mathbf{D}=\mathbf{0}$. Throughout we assume that $\mathbf{X}$ is strictly $\alpha$-stable and $\alpha \neq 1$. The probability density function of a stable distribution has no closed-form expression and moments with orders greater than or equal to $\alpha$ are not 
finite for the members of this class. The two aforementioned difficulties make statistical inference about the parameters of a stable distribution hard. However, a series of contributions has permitted inference about the parameters of univariate and multivariate stable distributions. For example, in the univariate case, maximum likelihood (ML) estimation was studied first by DuMouchel (1971) and then by Nolan [3]. Although the ML approach leads to an efficient estimate for samples of large size, it involves numerical complexities. A program, called STABLE uses a cubic spline interpolation of stable densities for this purpose; see Nolan [4]. STABLE estimates all four parameters of a stable distribution for $\alpha \geq$ 0.4. Sample quantile (SQ) technique is another approach proposed by McCulloch [5]. The results are simple and consistent estimators of all four parameters based on five sample quantiles. The empirical characteristic function (CF) is suggested by Kogon and Williams [6]. The CF and SQ methods work well but are not as efficient as the ML method. As the last approach considered here, $U$-statistics for the tail index and scale parameters of a univariate strictly stable distribution are introduced by Fan [7]. In multivariate case, the focus of interest is the spectral measure estimation. Among them, we refer to Nolan et al. [8], Pivato and Seco [9], Ogata [10], and Mohammadi et al. [11].

The structure of the paper is as follows. In Section 2, new estimators for the tail index and spectral measure of a strictly stable distribution are presented which is an extension of the $U$-statistic proposed by Fan [7] for the univariate case. A comprehensive simulation study is performed in Section 3 to compare the performance of the introduced estimators and the known estimators. Two real data sets are analyzed in this section to illustrate the performance of the proposed method.

\section{New Estimators}

This section consists of two subsections. Firstly, we propose an estimator for the tail index. Secondly, an estimator for the spectral measure is given.

2.1. Estimation of Tail Index. The main result of this section is given in Theorem 4, which gives $U$-statistic for the inverse of tail index of a strictly stable distribution. We present the main result in the light of Lemma 3 given as follows. The proofs are given in the Appendix.

Lemma 3. Let $\mathbf{X}=\left(X_{1}, \ldots, X_{d}\right)^{T}$ be a d-dimensional strictly stable random vector. Then, Var $\log \|\mathbf{X}\|$ is finite, where $\|\cdot\|$ denotes the Euclidean norm.

Theorem 4. Let $\mathbf{x}_{1}, \ldots, \mathbf{x}_{n}$ be a sequence of $n$ observations from a d-dimensional strictly stable random vector. Then

$$
U_{n}=\left(\begin{array}{l}
n \\
2
\end{array}\right)^{-1} \sum_{1 \leq i<j \leq n} H\left(\mathbf{x}_{i}, \mathbf{x}_{j}\right),
$$

where

$$
H\left(\mathbf{x}_{i}, \mathbf{x}_{j}\right)=\frac{\log \left\|\mathbf{x}_{i}+\mathbf{x}_{j}\right\|}{\log 2}-\frac{\log \left\|\mathbf{x}_{i}\right\|+\log \left\|\mathbf{x}_{j}\right\|}{2 \log 2}
$$

is the $U$-statistic for $1 / \alpha$.
As it is seen, from Theorem 4, the introduced $U$-statistic is an unbiased estimator for $1 / \alpha$. Hereafter, we write $\widehat{\alpha}_{\mathrm{MU}}=$ $1 / U_{n}$ as introduced estimator for $\alpha$. Here, subscript MU indicates that $\widehat{\alpha}_{\mathrm{MU}}$ is constructed based on multivariate $U$ statistic defined in Theorem 4 . It should be noted that when the true value of $\alpha$ is near two, the kernel given in (4) could be less than 0.5. So, $\widehat{\alpha}_{\mathrm{MU}}$ is greater than two. In this case, we set $\widehat{\alpha}_{\mathrm{MU}}=2$.

2.2. Spectral Measure Estimation. We use $\widehat{\alpha}_{\mathrm{MU}}$ to estimate an $m$-point discrete approximation to the exact spectral measure of the form

$$
\Gamma(\cdot)=\sum_{j=1}^{m} \gamma_{j} I_{s_{j}}(\cdot),
$$

where $\gamma_{j}$ is a mass at point $\mathbf{s}_{j}$ in the unit sphere $\mathbb{S}^{d}$ and $I_{\mathbf{s}_{j}}(\cdot)$ is an indicator function at point $\mathbf{s}_{j}$; for $j=1, \ldots, m$, see Byczkowski et al. [12]. To estimate $\Gamma(\cdot)$, we replace Definition 2 for a strictly $d$-dimensional stable random vector with

$$
\begin{aligned}
\varphi_{\mathbf{X}}(\mathbf{t}) & =-\sum_{j=1}^{m}\left|\left\langle\mathbf{t}, \mathbf{s}_{j}\right\rangle\right|^{\alpha}\left[1-i \operatorname{sgn}\left\langle\mathbf{t}, \mathbf{s}_{j}\right\rangle G\left(\alpha, \mathbf{t}, \mathbf{s}_{j}\right)\right] \gamma_{j}, \\
& =-\sum_{j=1}^{m} \psi\left(\mathbf{t}, \mathbf{s}_{j}, \alpha\right) \gamma_{j},
\end{aligned}
$$

where $G\left(\alpha, \mathbf{t}, \mathbf{s}_{j}\right)=\tan (\pi \alpha / 2)$ for $\alpha \neq 1$ and $-2 / \pi \log \left|\left\langle\mathbf{t}, \mathbf{s}_{j}\right\rangle\right|$ for $\alpha=1$. Define

$\Lambda$

$$
\begin{aligned}
& =\left(\begin{array}{cccc}
\psi\left(\mathbf{t}_{1}, \mathbf{s}_{1}, \alpha\right) & \psi\left(\mathbf{t}_{1}, \mathbf{s}_{2}, \alpha\right) & \cdots & \psi\left(\mathbf{t}_{1}, \mathbf{s}_{m}, \alpha\right) \\
\psi\left(\mathbf{t}_{2}, \mathbf{s}_{1}, \alpha\right) & \psi\left(\mathbf{t}_{2}, \mathbf{s}_{2}, \alpha\right) & \cdots & \psi\left(\mathbf{t}_{2}, \mathbf{s}_{m}, \alpha\right) \\
\vdots & \vdots & \ddots & \vdots \\
\psi\left(\mathbf{t}_{m}, \mathbf{s}_{1}, \alpha\right) & \psi\left(\mathbf{t}_{m}, \mathbf{s}_{2}, \alpha\right) & \cdots & \psi\left(\mathbf{t}_{m}, \mathbf{s}_{m}, \alpha\right)
\end{array}\right), \\
& \mathbf{V}=\left(-\log \varphi_{\mathbf{X}}\left(\mathbf{t}_{1}\right), \ldots,-\log \varphi_{\mathbf{X}}\left(\mathbf{t}_{m}\right)\right)^{T},
\end{aligned}
$$

where $\mathbf{t}_{j}=\left(t_{j 1}, \ldots, t_{j d}\right)^{T} \in \mathbb{S}^{d}$, for $j=1, \ldots, m$. Using (7) and (8), both sides of (6) are connected together through the following linear system:

$$
\mathrm{V}=\Lambda \gamma
$$

where $\gamma=\left(\gamma_{1}, \ldots, \gamma_{m}\right)^{T}$. Assuming that $\Lambda$ in (9) is nonsingular, then $\gamma=\Lambda^{-1} \mathbf{V}$. Hence, we estimate the vector of the masses as

$$
\widehat{\gamma}=\widehat{\Lambda}^{-1} \widehat{\mathbf{V}}
$$

where

$$
\widehat{\Lambda}
$$$$
=\left(\begin{array}{cccc}
\psi\left(\mathbf{t}_{1}, \mathbf{s}_{1}, \widehat{\alpha}_{\mathrm{MU}}\right) & \psi\left(\mathbf{t}_{1}, \mathbf{s}_{2}, \widehat{\alpha}_{\mathrm{MU}}\right) & \cdots & \psi\left(\mathbf{t}_{1}, \mathbf{s}_{m}, \widehat{\alpha}_{\mathrm{MU}}\right) \\
\psi\left(\mathbf{t}_{2}, \mathbf{s}_{1}, \widehat{\alpha}_{\mathrm{MU}}\right) & \psi\left(\mathbf{t}_{2}, \mathbf{s}_{2}, \widehat{\alpha}_{\mathrm{MU}}\right) & \cdots & \psi\left(\mathbf{t}_{2}, \mathbf{s}_{m}, \widehat{\alpha}_{\mathrm{MU}}\right) \\
\vdots & \vdots & \ddots & \vdots \\
\psi\left(\mathbf{t}_{m}, \mathbf{s}_{1}, \widehat{\alpha}_{\mathrm{MU}}\right) & \psi\left(\mathbf{t}_{m}, \mathbf{s}_{2}, \widehat{\alpha}_{\mathrm{MU}}\right) & \cdots & \psi\left(\mathbf{t}_{m}, \mathbf{s}_{m}, \widehat{\alpha}_{\mathrm{MU}}\right)
\end{array}\right),
$$ 


$$
\begin{aligned}
& \widehat{\mathbf{V}} \\
& =\left(-\log \frac{1}{n} \sum_{i=1}^{n} \exp \left\{i\left\langle\mathbf{t}_{1}, \mathbf{x}_{i}\right\rangle\right\}, \ldots,-\log \frac{1}{n} \sum_{i=1}^{n} \exp \left\{i\left\langle\mathbf{t}_{m}, \mathbf{x}_{i}\right\rangle\right\}\right)^{T},
\end{aligned}
$$

in which $\mathbf{x}_{i}$ is $i$-th vector observation in random sample of size $n$.

Due to the standard error of $\widehat{\mathbf{V}}$, we have two problems with direct use of (10). Firstly, $\widehat{\gamma}$ may be complex, and secondly, its real part may be quite negative. Since $\widehat{\Lambda}$ and $\widehat{\mathbf{V}}$ are complex while gamma is constrained to be real (and nonnegative), the Euclidean norm used by McCulloch [13] and Nolan et al. [8] must be replaced with the complex modulus to solve both problems in a novel way. For this, we use the nnls $(\cdot)$ library in the $\mathrm{R}$ package. In the next section, the estimated spectral measure $\widehat{\gamma}$, based on $\widehat{\alpha}_{\mathrm{MU}}$, is shown by $\widehat{\gamma}_{\mathrm{MU}}$. We note that another estimator of $\boldsymbol{\gamma}$ can be constructed by separating both of the real and imaginary parts in the structure of $\widehat{\mathbf{V}}$. But simulation results show that constructed estimator gives the same performance.

\section{Simulation Study}

This section is in three parts. Firstly, we study the performance of the proposed estimator with the known ones for estimating the tail index. Secondly, we compare the performance of the spectral measure estimator developed through the introduced tail index estimator with the known approaches. In the last subsection, we give a real data example to illustrate the efficiency of the proposed estimators.

3.1. Performance Analysis of the Tail Index Estimators. Here, we perform a simulation study to compare the performance of $\widehat{\alpha}_{\mathrm{MU}}$ and four other estimators for $\alpha$, including (1) $\widehat{\alpha}_{\mathrm{ML}},(2)$ $\widehat{\alpha}_{\mathrm{SQ}}$, (3) $\widehat{\alpha}_{\mathrm{CF}}$, and (4) $\widehat{\alpha}_{\mathrm{MM}}$. The first three competitors are ML, $\mathrm{SQ}$, and CF estimations for the tail index, respectively. Each of three competitors is obtained as $\widehat{\alpha}_{\mathrm{PROJ}}=1 / m \sum_{j=1}^{m} \widehat{\alpha}\left(\mathbf{u}_{j}\right)$ after projecting the $d$-dimensional stable random vector using $\left\langle\mathbf{u}_{j}, \mathbf{X}\right\rangle$. Here, $m$ is the number of masses, $\mathbf{u}_{j}=\left(u_{j 1}, \ldots, u_{j d}\right)^{T}$ is an arbitrary unit vector, and $\mathbf{X}$ is the $d$-dimensional stable random vector. It is worth noting that the first three competitors are computed by the help of STABLE software after projecting. The fourth estimator, that is, $\widehat{\alpha}_{M M}$, is the second estimator for tail index proposed by Mohammadi et al. [11]. We compare both the bias and root mean-squared error (RMSE) of estimators for 500 replications of samples of size $n=500$ and 5000 of a bivariate stable random vector generated by the method given in Modarres and Nolan [14]. We use two settings for discrete spectral measure with $m=8$ masses, including $\gamma_{1}=(0.1,0.2,0.3,0.4,0.1,0.2,0.3,0.4)^{T}$ and $\gamma_{2}=$ $(0,0.1,0.7,0.3,0.7,0.3,0.7,0.1)^{T}$. In both cases, masses are concentrated on points $\mathbf{s}_{j}=(\cos (2 \pi(j-1) / m), \sin (2 \pi(j-$ $1) / m))^{T}$ for $j=1, \ldots, 8$. In the first case that data are coming from a stable distribution with $\gamma_{1}$, we generate $\mathbf{t}_{j}$ from a uniform distribution on the unit sphere $\mathbb{S}^{d}$. For the second case, we set $\mathbf{t}_{j}=\mathbf{s}_{j}$. Biases and RMSEs for $\alpha=(0.1: 0.1: 0.9,0.95,1.05,1.1: 0.1: 1.9,1.95,2)$ are shown in Figures 1 and 2 . As Figure 1 shows, when $\gamma_{1}=$ $(0.1,0.2,0.3,0.4,0.1,0.2,0.3,0.4)^{T}$, we observe that $\widehat{\alpha}_{\mathrm{MU}}$ is more efficient than $\widehat{\alpha}_{\mathrm{SQ}}$ for $n=5000$. Also, it works better than $\widehat{\alpha}_{\mathrm{MM}}$ in terms of RMSE (for $\alpha \leq 1.8$ ). Based on Figure 2, when $\gamma_{2}=(0,0.1,0.7,0.3,0.7,0.3,0.7,0.1)^{T}$, we observe that $\widehat{\alpha}_{\mathrm{MU}}$ is more efficient than other methods when $\alpha<1.4$ and $n=5000$ in the sense of RMSE. Also, when $\alpha<1.7$ and $n=500, \widehat{\alpha}_{\mathrm{MU}}$ is more efficient than $\widehat{\alpha}_{\mathrm{SQ}}, \widehat{\alpha}_{\mathrm{CF}}$, and $\widehat{\alpha}_{\mathrm{MM}}$ with respect to RMSE.

3.2. Performance Analysis of the Spectral Measure Estimators. Here, we compare the performance of the estimator for masses of spectral measure $\gamma=\left(\gamma_{1}, \ldots, \gamma_{m}\right)^{T}$ constructed based on $U$-statistic, $\widehat{\gamma}_{\mathrm{MU}}$ with the other four known estimators for the spectral measure. The competitors are three types of estimators for $\gamma$ based on empirical characteristic function method: (1) $\widehat{\gamma}_{\text {MLE-cf }} ;(2) \widehat{\gamma}_{\text {SQ-cf }} ;$ (3) $\widehat{\gamma}_{\text {CF-cf }}$; and (4) Mohammadi et al. [11] estimator for $\gamma, \widehat{\gamma}_{\mathrm{MM}}$. For computing $\widehat{\gamma}_{\text {MLE-cf }}, \widehat{\gamma}_{\text {SQ-cf }}$, and $\widehat{\gamma}_{\text {CF-cf }}$, we use command mvstable.fit(x, nspectral, method1d, method $2 \mathrm{~d}$, param) in the STABLE program, where $\mathbf{x}$ is data vector, nspectral is number of spectral measure masses, method1d is the method to use for estimating parameters of univariate stable distribution, that is, MLE, SQ, and CF (corresponding codes in STABLE are 1, 2, and 3, respectively), method $2 \mathrm{~d}$ is the method to use for estimating parameters of bivariate stable distribution (we set method $2 \mathrm{~d}=2$ which corresponds to empirical characteristic function approach, cf), and param refers to kind of parameterization. Here, we set param $=1$ since we are using the characteristic function in (2). More information about the first three competitors is given in Robust Analysis Inc. [15]. The estimators $\widehat{\gamma}_{\text {MLE-cf }}, \widehat{\gamma}_{\text {SQ-cf }}$, $\widehat{\gamma}_{\mathrm{CF}-\mathrm{cf}}$, and $\widehat{\gamma}_{\mathrm{MM}}$ are obtained by substituting $\widehat{\alpha}_{\mathrm{ML}}, \widehat{\alpha}_{\mathrm{SQ}}$, $\widehat{\alpha}_{\mathrm{CF}}$, and $\widehat{\alpha}_{\mathrm{MM}}$ into (7) and then solving linear system (10), respectively. Comparisons are based on the RMSE of $\widehat{\gamma}_{j}$, for $j=1, \ldots, m$, which is defined as $\sqrt{1 / N \sum_{i=1}^{N}\left(\widehat{\gamma}_{i j}-\gamma_{i j}\right)^{2}}$, where $N$ is the number of iterations and $\widehat{\gamma}_{i j}$ is the estimation of $j$ th component of $\widehat{\gamma}$ at ith iteration. We consider five scenarios for the structure of discrete spectral measure as follows.

(1) Independent case: $\gamma=(1 / 4,0,1 / 4,0,1 / 4,0,1 / 4,0)^{T}$.

(2) Symmetric case: $\gamma=(0.1,0.2,0.3,0.4,0.1,0.2,0.3$, $0.4)^{T}$.

(3) Uniform case: $\gamma=(1 / 8,1 / 8,1 / 8,1 / 8,1 / 8,1 / 8,1 / 8,1 /$ $8)^{T}$.

(4) Triangle case: $\boldsymbol{\gamma}=(0,0.1,0.7,0.3,0.7,0.3,0.7,0.1)^{T}$.

(5) Exchangeable case: $\gamma=(0.1,0.2,0.1,0.4,0.3,0.2,0.3$, $0.4)^{T}$.

We note that the first and the third scenarios above are similar to Examples 2 and 1 of Nolan et al. [8], respectively. The fourth scenario is called Triangle since corresponding density contour plot is similar to a triangle. For each of the above five scenarios, we arrange the settings of simulation as $m=8, \alpha=1.25 ; 1.75 ; n=2000 ; 5000$ ( $n$ is sample size), 


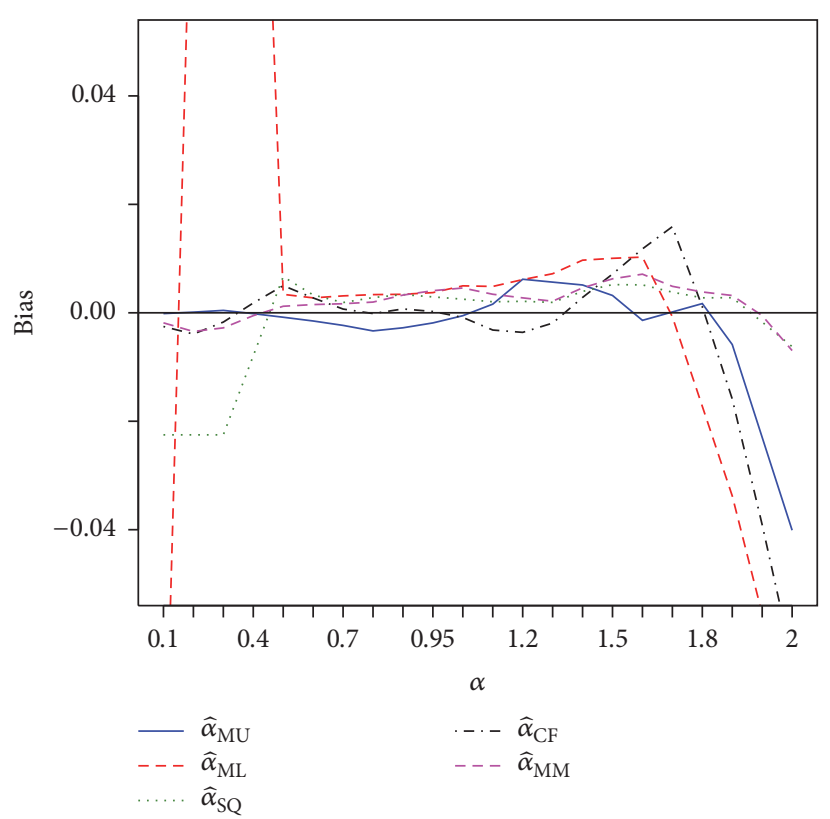

(a)

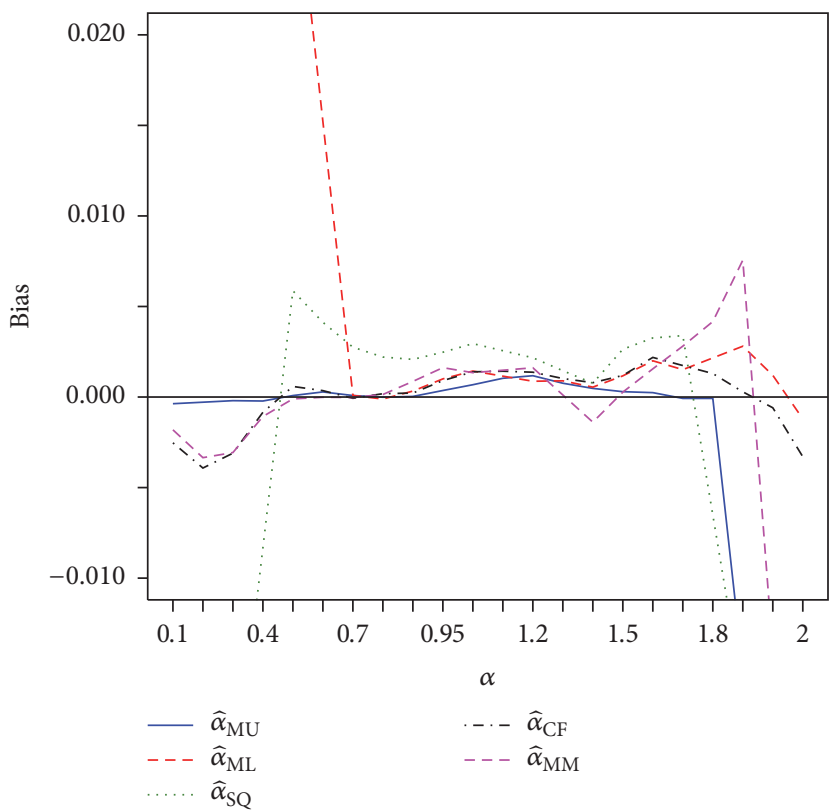

(c)

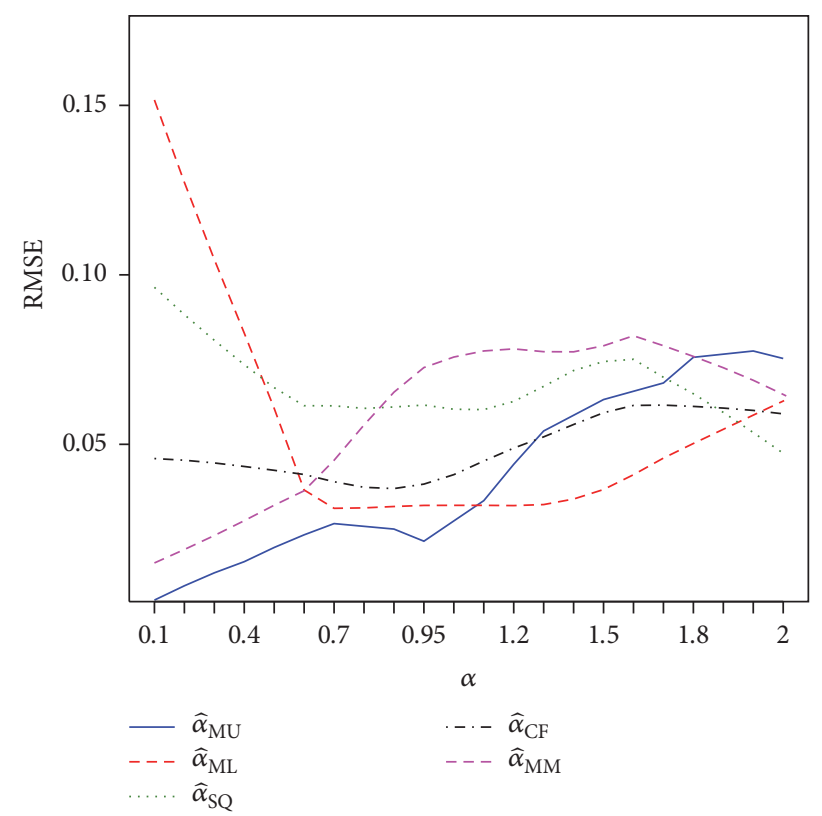

(b)

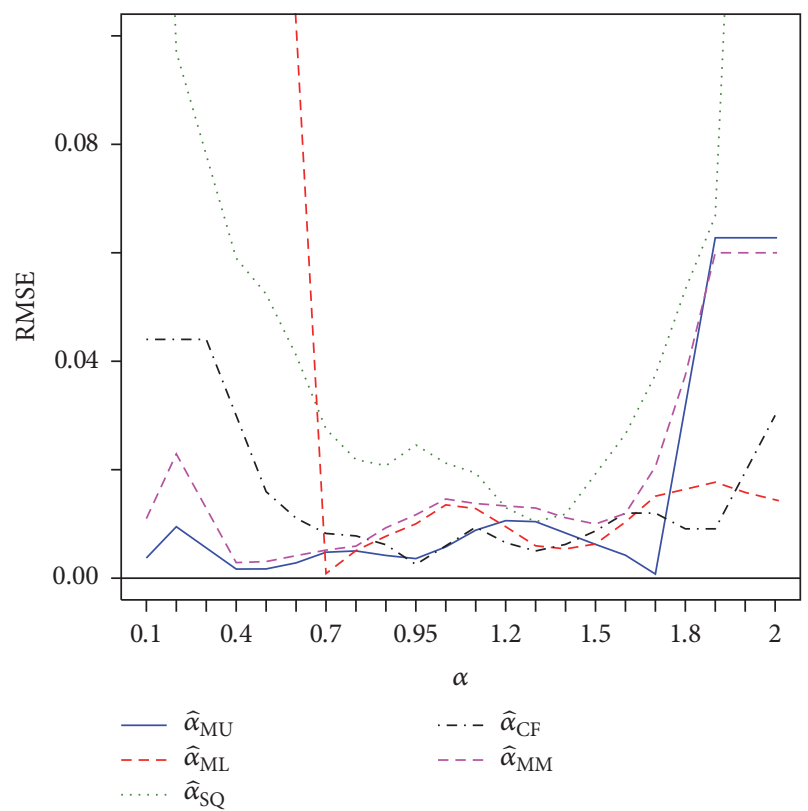

(d)

FIGURE 1: Biases and RMSEs of estimators when data are generated from a strictly stable distribution with discrete spectral measure $\gamma_{1}=$ $(0.1,0.2,0.3,0.4,0.1,0.2,0.3,0.4)^{T}$. (a) Bias when $n=500$, (b) RMSE when $n=500$, (c) bias when $n=5000$, and (d) RMSE when $n=5000$.

and $N=500$. It should be noted that masses are located at $\mathbf{s}_{j}=(\cos (2 \pi(j-1) / m), \sin (2 \pi(j-1) / m))^{T}$, for $j=1, \ldots, m$, and components of $\mathbf{t}_{j}=\left(t_{j 1}, \ldots, t_{j d}\right)^{T}$ are generated from a uniform distribution on the unit sphere $\mathbb{S}^{d}$. The results of simulations are given in Figures 3-6. As it is seen, $\widehat{\gamma}_{\mathrm{MU}}$ shows better performance than $\widehat{\gamma}_{\mathrm{MM}}$.

3.3. Real Data Analysis. Here, we give two examples. In the first example, adjusted daily log-return (in percent) for the 30 stocks at the Dow Jones index is collected between January 3, 2000, and December 31, 2004. The log-return percent of 1247 closing prices has been computed for AXP (American Express Company) and MRK (Merck \& Co. Inc.) stocks after multiplying the daily log-return by 100 ; see Nolan [16]. The scatter plot of AXP and MRK stocks log-return percent values, $\mathbf{X}=(\mathrm{AXP}, \mathrm{MRK})^{T}$, is shown in Figure 7 . We use a bivariate $\alpha$-stable distribution with $m=12$ points of masses for spectral measure addressed by $\mathbf{s}_{j}=(\cos (2 \pi(j-$ $1) / m), \sin (2 \pi(j-1) / m))^{T}$, for $j=1, \ldots, 12$. We estimate the 


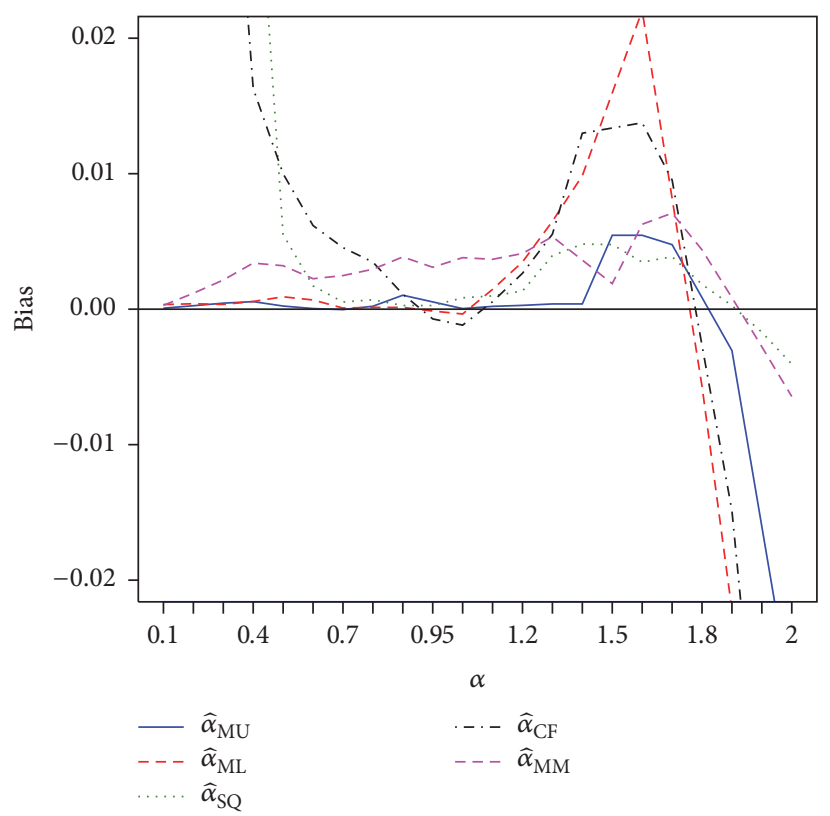

(a)

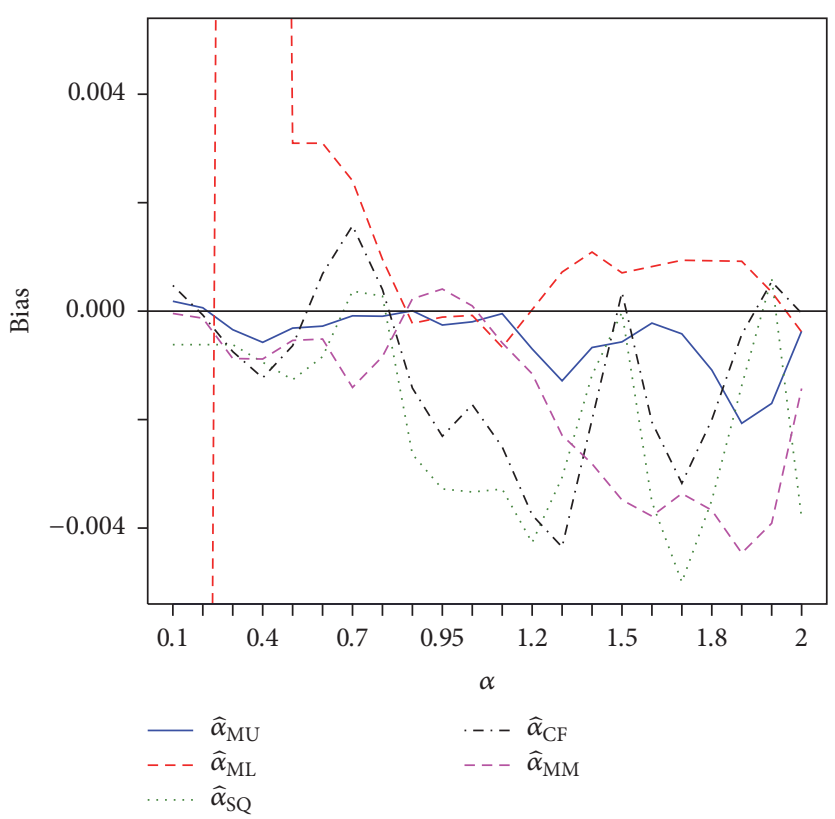

(c)

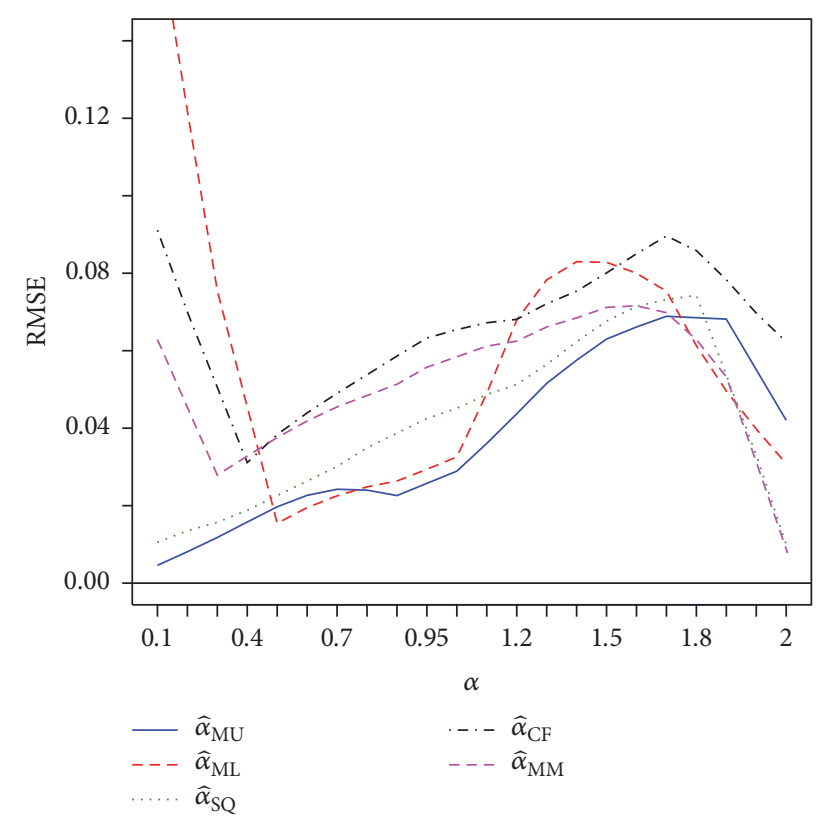

(b)

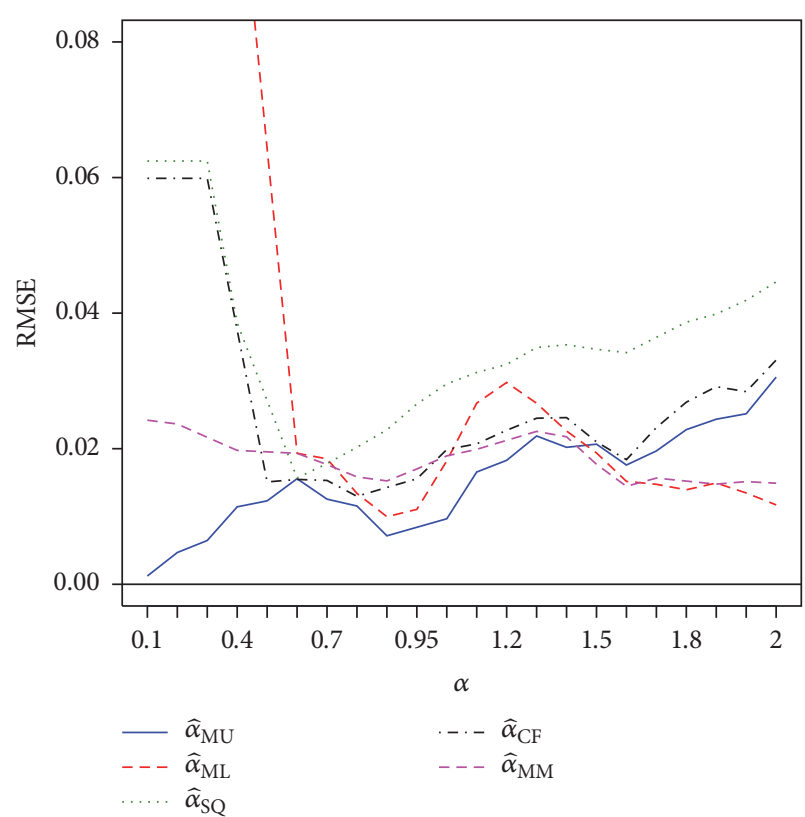

(d)

FIGURE 2: Biases and RMSEs of estimators when data are generated from a strictly stable distribution with discrete spectral measure $\gamma_{2}=$ $(0,0.1,0.7,0.3,0.7,0.3,0.7,0.1)^{T}$. (a) Bias when $n=500$, (b) RMSE when $n=500$, (c) bias when $n=5000$, and (d) RMSE when $n=5000$.

location parameter as $\widehat{\mu}_{\text {ML-cf }}=(-3.438 E-07,-2.402 E-$ $07)^{T}$. So, a strictly $\alpha$-stable distribution is fitted to the $\mathbf{Y}=$ $\left(\mathbf{X}-\widehat{\mu}_{\mathrm{ML}-\mathrm{cf}}\right)^{T}$. For this, we set $\mathbf{t}_{j}=\mathbf{s}_{j}$, for $j=1, \ldots, 12$. Table 1 shows the results for modelling data through five methods. We note that estimated tail indices are $\widehat{\alpha}_{\mathrm{MU}}=$ $1.581, \widehat{\alpha}_{\mathrm{MM}}=1.734, \widehat{\alpha}_{\mathrm{ML}-\mathrm{cf}}=1.618, \widehat{\alpha}_{\mathrm{SQ}-\mathrm{cf}}=1.493$, and $\widehat{\alpha}_{\text {CF-cf }}=1.723$. As it is seen, estimated tail indices through estimators $\widehat{\alpha}_{\mathrm{MU}}$ and $\widehat{\alpha}_{\mathrm{ML}-\mathrm{cf}}$ are closer together than the other estimators. In the second example, we focus on the cubic-root of the monthly average of river discharge. We choose discharge of the Odra and Wisla rivers in Poland during 1901 to 1986 (raw data are in $\mathrm{m}^{3} / \mathrm{s}$. They are available at https://nelson.wisc.edu/sage/data-and-models/riverdata/). The scatter plot for cubic-root of Odra river discharge versus cubic-root of Wisla river discharge is shown in Figure 8. Setting $m=8, \boldsymbol{s}_{j}=(\cos (2 \pi(j-1) / m), \sin (2 \pi(j-1) / m))^{T}$, and 

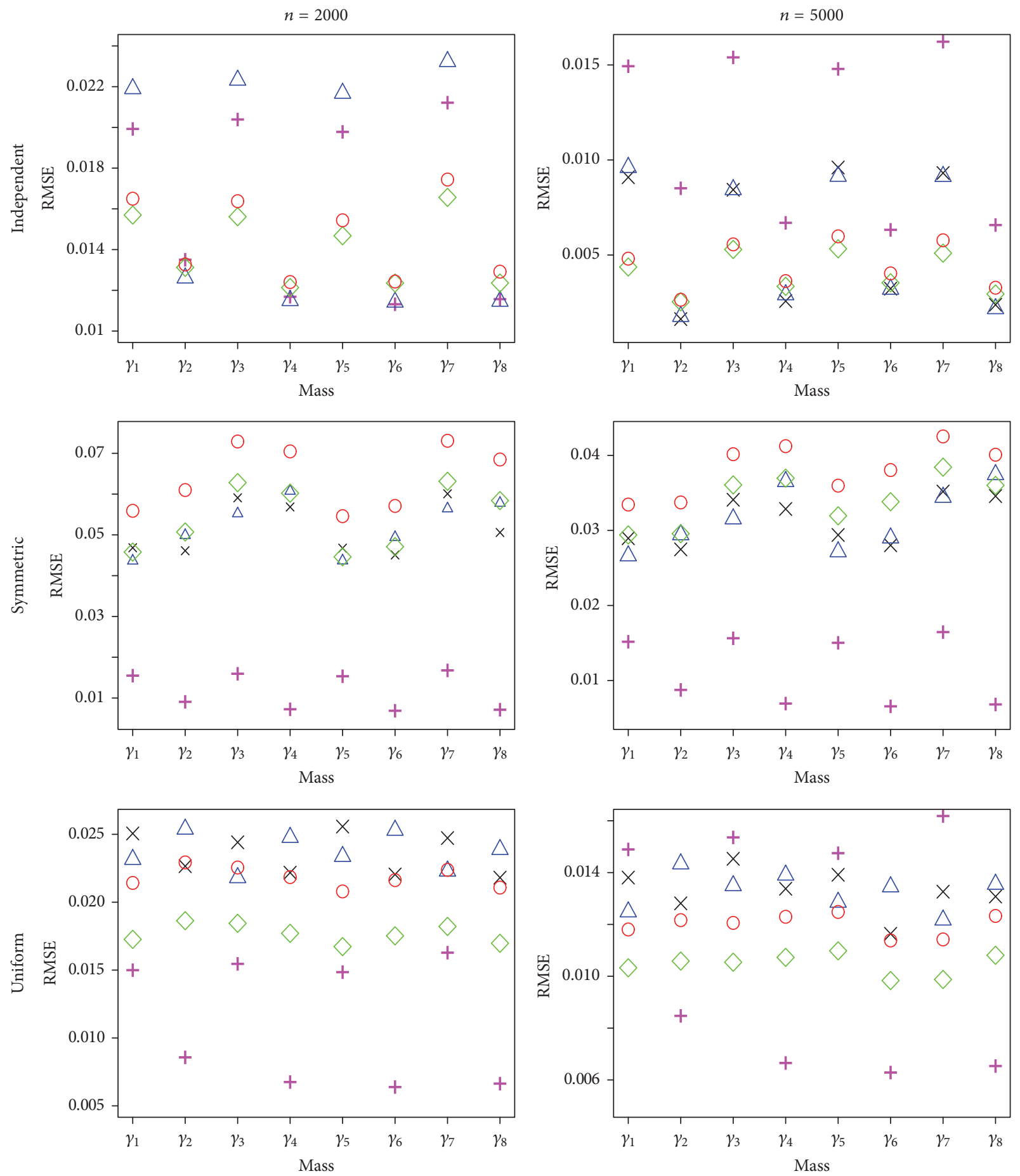

FIGURE 3: RMSEs of $\widehat{\gamma}$ under different scenarios when $\alpha=1.25$. We use the following symbol scheme: $\diamond$ for $\widehat{\gamma}_{\mathrm{UM}}, \mathrm{O}$ for $\widehat{\gamma}_{\mathrm{MM}},+$ for $\widehat{\gamma}_{\mathrm{ML}-\mathrm{cf}}, \times$ for $\widehat{\gamma}_{\mathrm{SQ}-\mathrm{cf}}$, and $\triangle$ for $\widehat{\gamma}_{\mathrm{CF}-\mathrm{cf}}$.

$\mathbf{t}_{j}=\mathbf{s}_{j}$, for $j=1, \ldots, 8$, we obtain $\widehat{\boldsymbol{\mu}}_{\mathrm{ML}-\mathrm{cf}}=(7.9837,9.9947)^{T}$. After fitting a strictly $\alpha$-stable distribution to the shifted data, results for estimating spectral measure are given in Table 2. Estimated tail indices are $\widehat{\alpha}_{\mathrm{MU}}=1.860, \widehat{\alpha}_{\mathrm{MM}}=1.312$, $\widehat{\alpha}_{\mathrm{ML}-\mathrm{cf}}=1.813, \widehat{\alpha}_{\mathrm{SQ}-\mathrm{cf}}=1.936$, and $\widehat{\alpha}_{\mathrm{CF}-\mathrm{cf}}=1.962$. Based on results given in Table 2, estimated masses through estimators $\widehat{\gamma}_{\mathrm{MU}}, \widehat{\gamma}_{\mathrm{ML}-\mathrm{cf}}$, and $\widehat{\gamma}_{\mathrm{SQ}-\mathrm{cf}}$ are closer together than the other estimators. We compare here $\widehat{\gamma}_{\mathrm{MU}}$ with $\widehat{\gamma}_{\mathrm{ML}-\mathrm{cf}}$ and $\widehat{\gamma}_{\mathrm{SQ}-\mathrm{cf}}$ since the latter estimators are among the best estimators for the masses as shown in the previous subsection. 

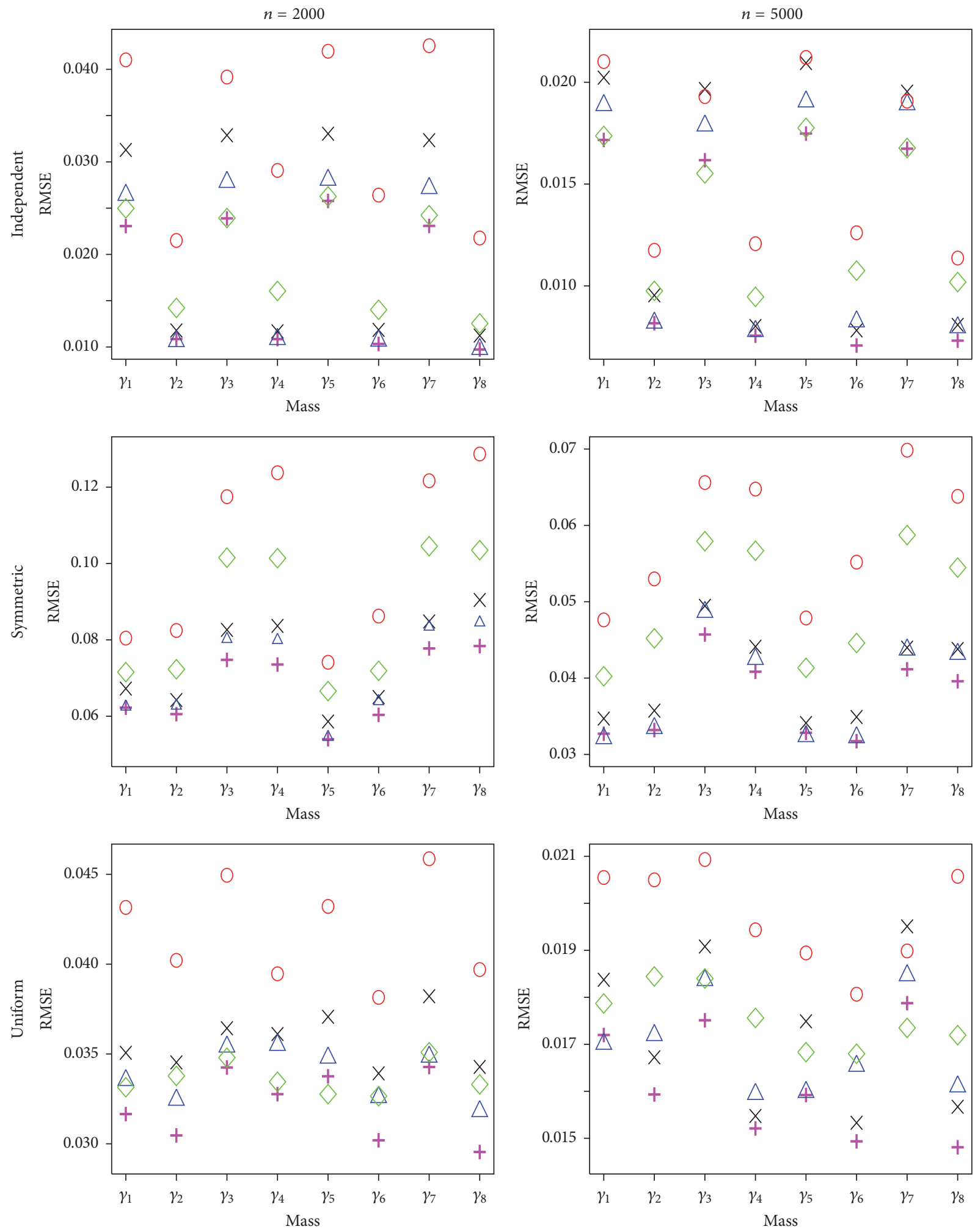

FIGURE 4: RMSEs of $\hat{\gamma}$ under different scenarios when $\alpha=1.75$. We use the following symbol scheme: $\diamond$ for $\widehat{\gamma}_{\text {UM }}, \bigcirc$ for $\widehat{\gamma}_{\text {MM }},+$ for $\widehat{\gamma}_{\text {ML-cf }}, \times$ for $\widehat{\gamma}_{\text {SQ-cf }}$, and $\Delta$ for $\widehat{\gamma}_{\text {CF-cf }}$. 

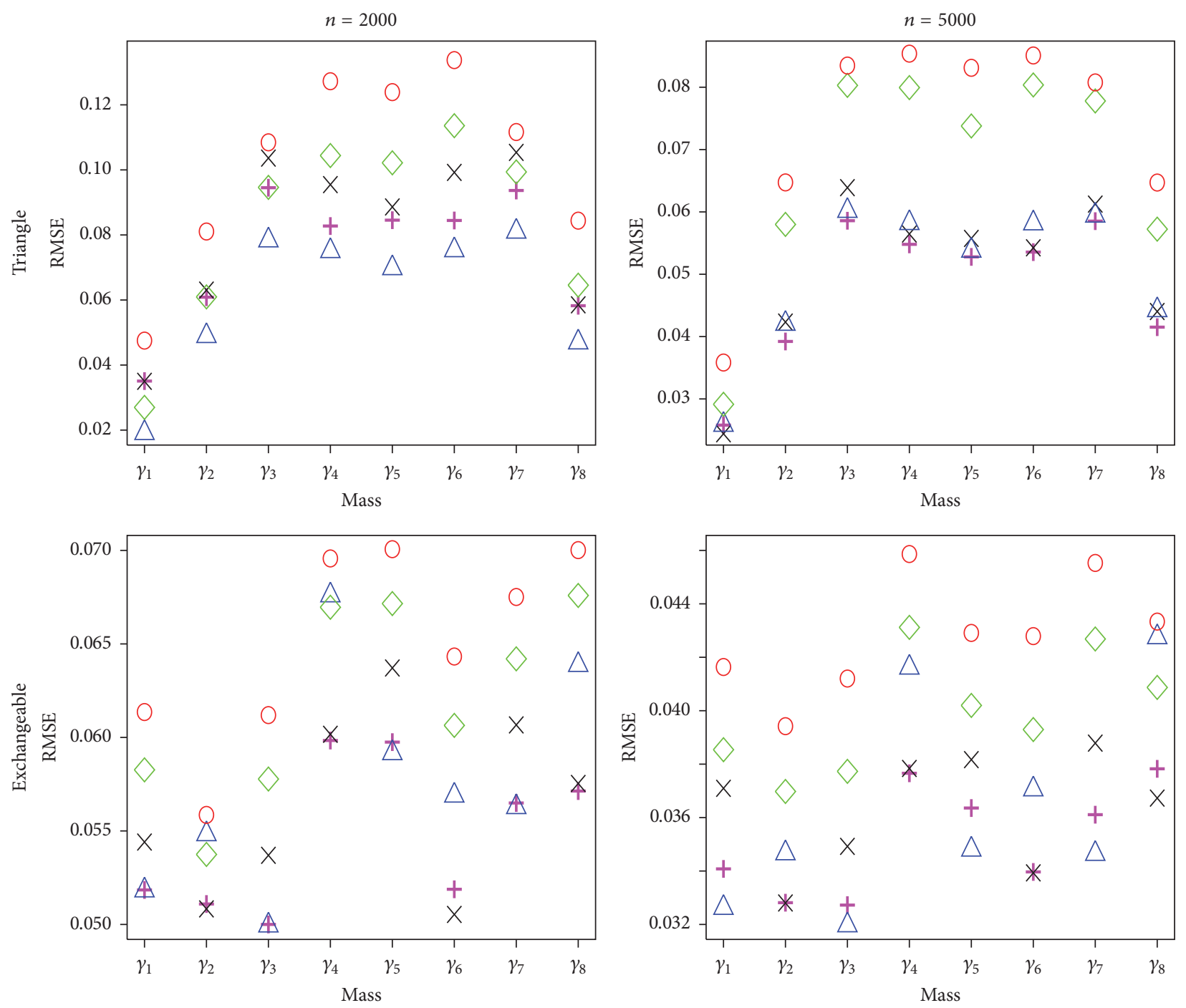

FigURE 5: RMSEs of $\hat{\gamma}$ under different scenarios when $\alpha=1.25$. We use the following symbol scheme: $\diamond$ for $\widehat{\gamma}_{\mathrm{UM}}$, O for $\widehat{\gamma}_{\mathrm{MM}}$, + for $\widehat{\gamma}_{\mathrm{ML}-\mathrm{cf}}, \times$ for $\widehat{\gamma}_{\mathrm{SQ}-\mathrm{cf}}$, and $\triangle$ for $\widehat{\gamma}_{\mathrm{CF}-\mathrm{cf}}$.

\section{Conclusion}

We compare the performance of the introduced $U$-statistic for the tail index with the well-known methods, including maximum likelihood, empirical characteristic function, sample quantile, and that introduced in Mohammadi et al. [11] through a simulation study. In the sense of root meansquared error, it is proved that proposed tail index estimator always outperforms Mohammadi et al. [11] and SQ methods when $\alpha \leq 1.4$. This is while ML and CF methods show better performance than the proposed estimator for large $\alpha$, say $\alpha>1.4$ in terms of root mean-squared error. Simulation studies for estimating the discrete spectral measure $\gamma$ under five scenarios prove that estimator of $\gamma$ based on introduced $U$-statistic shows, in terms of root mean-squared error, better performance than Mohammadi et al. [11] estimator. Analysis of two sets of real data reveals that estimator of the tail index and $\gamma$ based on $U$-statistic shows expedient performance. As some possible future works, firstly, we aim to introduce a $U$-statistic for the case of a nonzero location parameter. Secondly, we look for methodology possibly based on a $U$ statistic, to estimate tail, masses, and location parameters simultaneously. Finally, recalling that the approach employed in this work is based on characteristic function, the discrete spectral measure using $\widehat{\alpha}_{\mathrm{MU}}$ can be estimated through projection approach.

\section{Appendix}

Proof of Lemma 3. We show that $E\left(\log ^{2}\|\mathbf{X}\|\right)<\infty$. Suppose $d=2$ and $p^{-}=P(\|\mathbf{X}\| \leq 1), p^{+}=1-p^{-}, p^{--}=P\left(X_{1}<\right.$ $\left.0, X_{2}<0\right), p^{-+}=P\left(X_{1}<0, X_{2}>0\right), p^{+-}=P\left(X_{1}>0, X_{2}<\right.$ $0)$, and $p^{++}=P\left(X_{1}>0, X_{2}>0\right)$. So

$$
\begin{aligned}
E\left(\log ^{2}\|\mathbf{X}\|\right)= & E\left(\log ^{2}\|\mathbf{X}\| \mid\|\mathbf{X}\|<1\right) p^{-} \\
& +E\left(\log ^{2}\|\mathbf{X}\| \mid\|\mathbf{X}\| \geq 1\right) p^{+}
\end{aligned}
$$



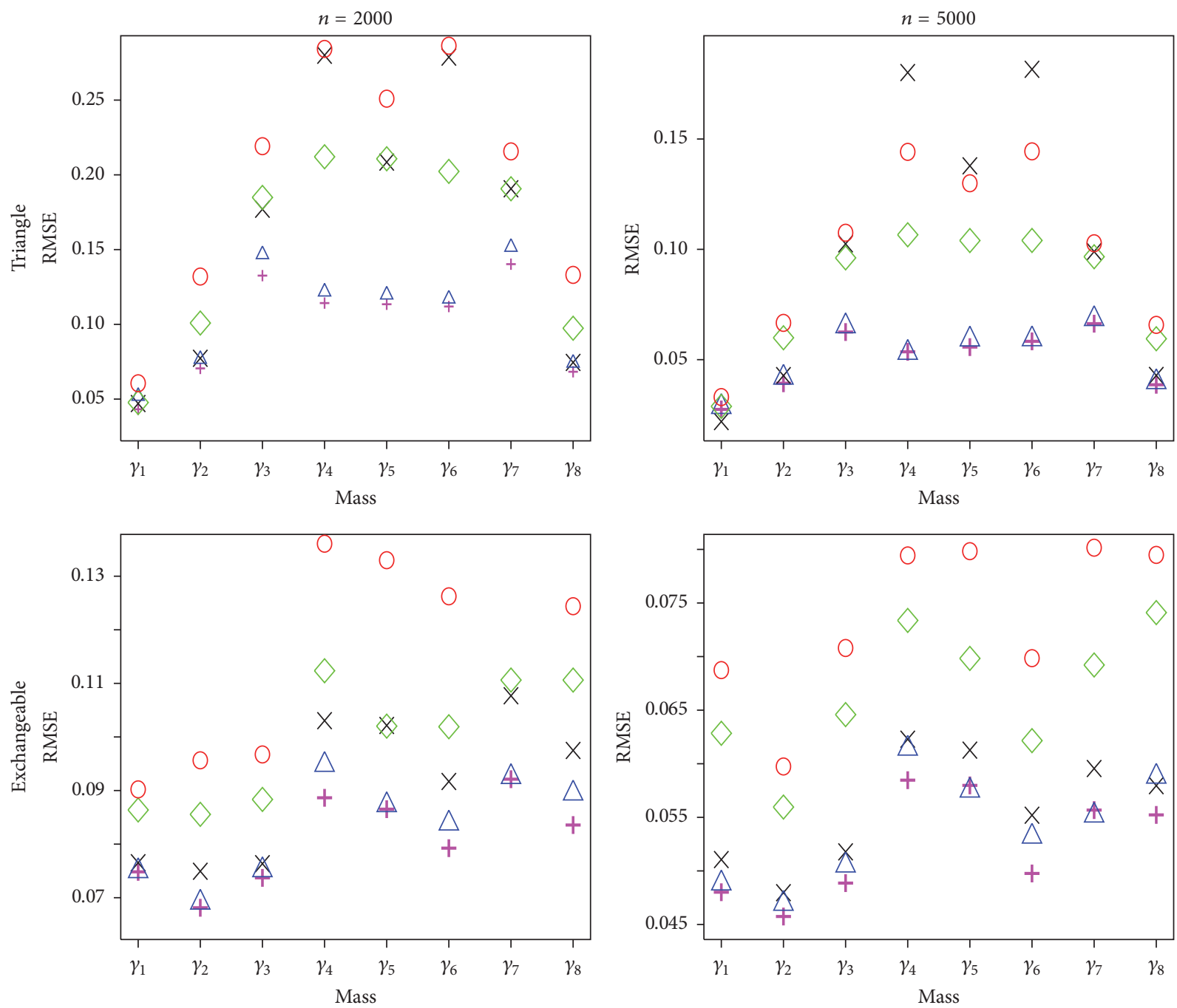

FIGURE 6: RMSEs of $\hat{\gamma}$ under different scenarios when $\alpha=1.75$. We use the following symbol scheme: $\diamond$ for $\widehat{\gamma}_{\mathrm{UM}}$, O for $\widehat{\gamma}_{\mathrm{MM}}$, + for $\widehat{\gamma}_{\mathrm{ML}-\mathrm{cf}}, \times$ for $\widehat{\gamma}_{\mathrm{SQ}-\mathrm{cf}}$, and $\triangle$ for $\widehat{\gamma}_{\mathrm{CF}-\mathrm{cf}}$.

TABLE 1: Estimation results after fitting a strictly bivariate $\alpha$-stable distribution to AXP and MRK stocks data.

\begin{tabular}{lcccccccccccc}
\hline Estimator & $\widehat{\gamma}_{1}$ & $\widehat{\gamma}_{2}$ & $\widehat{\gamma}_{3}$ & $\widehat{\gamma}_{4}$ & $\widehat{\gamma}_{5}$ & $\widehat{\gamma}_{6}$ & $\widehat{\gamma}_{7}$ & $\widehat{\gamma}_{8}$ & $\widehat{\gamma}_{9}$ & $\widehat{\gamma}_{10}$ & $\widehat{\gamma}_{11}$ & $\widehat{\gamma}_{12}$ \\
\hline$\widehat{\gamma}_{\text {MU }}$ & 0.396 & 0.070 & 0.077 & 0.433 & 0.087 & 0 & 0.231 & 0.378 & 0 & 0.439 & 0.078 & 0 \\
$\widehat{\gamma}_{\text {MM }}$ & 0.373 & 0 & 0 & 0.463 & 0.021 & 0 & 0.333 & 0.479 & 0 & 0.563 & 0.100 & 0 \\
$\widehat{\gamma}_{\text {ML-cf }}$ & 0.338 & 0.156 & 0.162 & 0.412 & 0 & 0 & 0.490 & 0.206 & 0.055 & 0.425 & 0.122 & 0 \\
$\widehat{\gamma}_{\text {SQ-cf }}$ & 0.232 & 0.216 & 0.089 & 0.404 & 0.012 & 0 & 0.480 & 0.115 & 0.177 & 0.254 & 0.156 & 0 \\
$\widehat{\gamma}_{\text {CF-cf }}$ & 0.421 & 0.129 & 0.237 & 0.434 & 0 & 0 & 0.537 & 0.209 & 0 & 0.527 & 0.089 & 0 \\
\hline
\end{tabular}

TABLE 2: Estimation results after fitting a strictly bivariate $\alpha$-stable distribution to Odra and Wisla discharge data.

\begin{tabular}{lccccccc}
\hline Estimator & $\widehat{\gamma}_{1}$ & $\widehat{\gamma}_{2}$ & $\widehat{\gamma}_{3}$ & $\widehat{\gamma}_{4}$ & $\widehat{\gamma}_{5}$ & $\widehat{\gamma}_{6}$ & $\widehat{\gamma}_{7}$ \\
\hline$\widehat{\gamma}_{\mathrm{MU}}$ & 0.006 & 1.459 & 0.498 & 0 & 0 & 0 & 0 \\
$\widehat{\gamma}_{\mathrm{MM}}$ & 0 & 0.800 & 0.257 & 0 & 0.026 & 0.483 & 0.165 \\
$\widehat{\gamma}_{\mathrm{ML-cf}}$ & 0 & 1.380 & 0.508 & 0 & 0 & 0 & 0 \\
$\widehat{\gamma}_{\text {SQ-cf }}$ & 0 & 1.490 & 0.532 & 0 & 0 & 0 & 0 \\
$\widehat{\gamma}_{\text {CF-cf }}$ & 0 & 0.196 & 0.199 & 0 & 0.009 & 1.283 & 0 \\
\hline
\end{tabular}




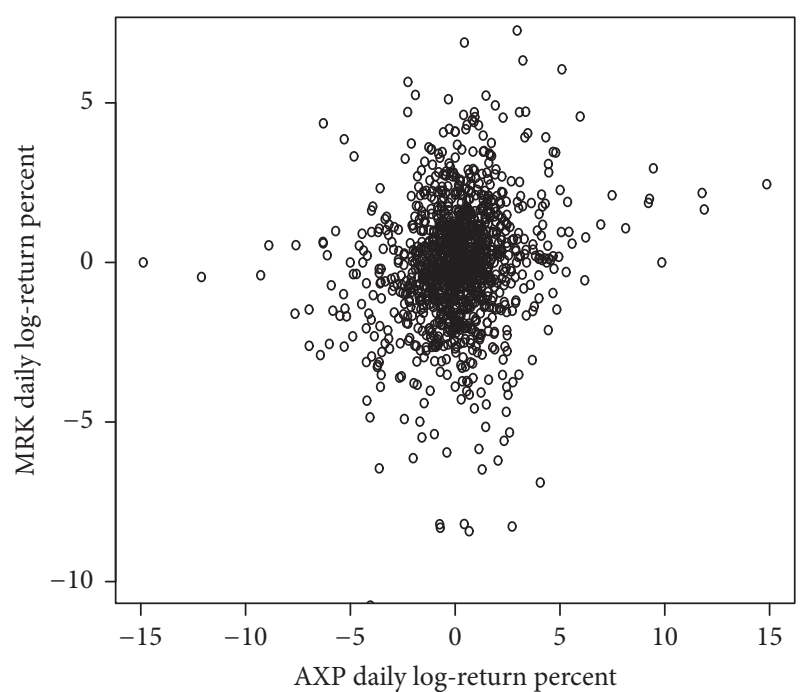

FIGURE 7: Scatter plot for AXP versus MRK daily log-return percent, $\mathbf{X}=(\mathrm{AXP}, \mathrm{MRK})^{T}$.

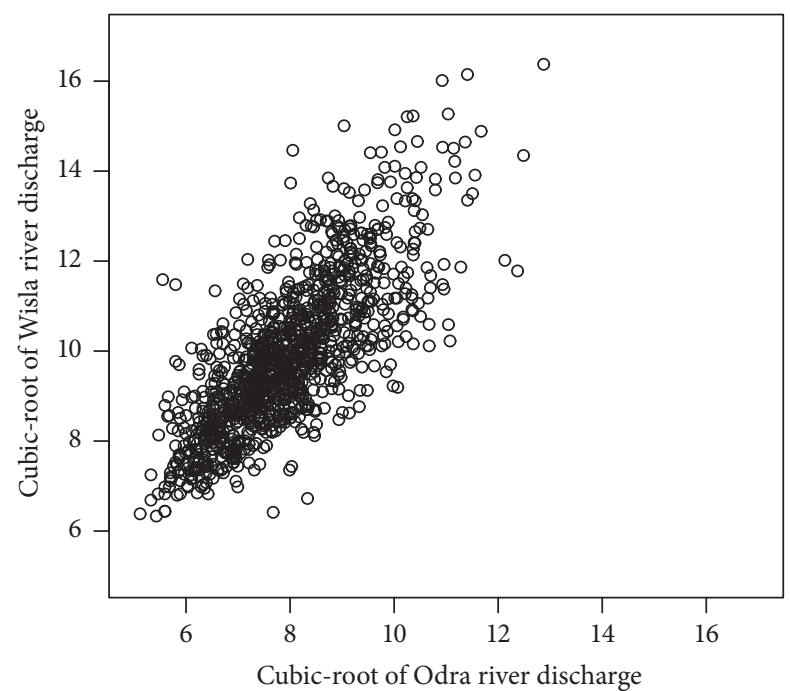

FIGURE 8: Scatter plot for cubic-root of Odra and Wisla rivers discharge.

$$
\begin{aligned}
\leq & E\left(\log ^{2}|X| \mid\|\mathbf{X}\|<1\right) p^{-} \\
& +E\left(\log ^{2}\|\mathbf{X}\| \mid\|\mathbf{X}\| \geq 1\right) p^{+} \\
\leq & E\left(\log ^{2}|X|\right) \\
& +E\left(\log ^{2}\|\mathbf{X}\| \mid\|\mathbf{X}\| \geq 1\right),
\end{aligned}
$$

where $X$, in the above, denotes one of the components of vector $\mathbf{X}$. It should be noted that inequality $E\left(\log ^{2}\|\mathbf{X}\| \mid\|\mathbf{X}\|<\right.$ $1) \leq E\left(\log ^{2}|X| \mid\|\mathbf{X}\|<1\right)$ holds irrespective of $d$. On the other hand,

$$
\begin{aligned}
& E\left(\log ^{2}\|\mathbf{X}\| \mid\|\mathbf{X}\| \geq 1\right) \\
& \quad=E\left(\log ^{2}\|\mathbf{X}\| \mid\|\mathbf{X}\| \geq 1, X_{1}<0, X_{2}<0\right) p^{--}
\end{aligned}
$$

$$
\begin{aligned}
& +E\left(\log ^{2}\|\mathbf{X}\| \mid\|\mathbf{X}\| \geq 1, X_{1}<0, X_{2}>0\right) p^{-+} \\
& +E\left(\log ^{2}\|\mathbf{X}\| \mid\|\mathbf{X}\| \geq 1, X_{1}>0, X_{2}<0\right) p^{+-} \\
& +E\left(\log ^{2}\|\mathbf{X}\| \mid\|\mathbf{X}\| \geq 1, X_{1}>0, X_{2}>0\right) p^{++} \\
& \leq E\left(\log ^{2}\left|X_{1}+X_{2}\right| \mid\|\mathbf{X}\| \geq 1, X_{1}<0, X_{2}<0\right) \\
& \cdot p^{--} \\
& +E\left(\log ^{2}\left|-X_{1}+X_{2}\right| \mid\|\mathbf{X}\| \geq 1, X_{1}<0, X_{2}>0\right) \\
& \cdot p^{-+} \\
& +E\left(\log ^{2}\left|X_{1}-X_{2}\right| \mid\|\mathbf{X}\| \geq 1, X_{1}>0, X_{2}<0\right) \\
& \cdot p^{+-} \\
& +E\left(\log ^{2}\left|X_{1}+X_{2}\right| \mid\|\mathbf{X}\| \geq 1, X_{1}>0, X_{2}>0\right) \\
& \cdot p^{++} \leq 2 E\left(\log ^{2}\left|X_{1}+X_{2}\right|\right) \\
& +2 E\left(\log ^{2}\left|X_{1}+X_{2}\right|\right) .
\end{aligned}
$$

Thus,

$$
\begin{aligned}
E\left(\log ^{2}\|\mathbf{X}\|\right) \leq & E\left(\log ^{2}|X|\right)+2 E\left(\log ^{2}\left|X_{1}+X_{2}\right|\right) \\
& +2 E\left(\log ^{2}\left|X_{1}-X_{2}\right|\right) .
\end{aligned}
$$

Generally, for $d \geq 2$, one can write

$$
\begin{aligned}
& E\left(\log ^{2}\|\mathbf{X}\|\right) \\
& \leq E\left(\log ^{2}|X|\right) \\
& \quad+\sum_{i=0}^{d}\left(\begin{array}{c}
d \\
i
\end{array}\right) E\left(\log ^{2}\left|-\sum_{j=1}^{i} X_{j}+X_{i+1}+\cdots+X_{d}\right|\right),
\end{aligned}
$$

where we adopt this convention that $\sum_{j=1}^{0} X_{j}=0$. Let $S(\alpha, \beta, \sigma, \mu=0)$ stands for a univariate strictly stable random variable with tail index $\alpha$, scale parameter $\sigma$, and skewness parameter $\beta$. It is well known that if $\mathbf{X}=\left(X_{1}, \ldots, X_{d}\right)^{T}$ is an $\alpha$-stable random vector, then any linear combination of its components such as $\left\langle\mathbf{b}_{i}, \mathbf{X}\right\rangle=\sum_{j=1}^{d} b_{i j} X_{j}$, for $\mathbf{b}_{i}=(\overbrace{-1, \ldots,-1}^{i}, \overbrace{1, \ldots, 1}^{d-i})^{T}$, follows a stable distribution with tail index $\alpha$,

$$
\begin{aligned}
& \sigma_{i}=\left(\int_{\mathbb{S}^{d}}\left|\left\langle\mathbf{b}_{\mathbf{i}}, \mathbf{s}\right\rangle\right|^{\alpha} \Gamma(d \mathbf{s})\right)^{1 / \alpha}, \\
& \beta_{i}=\frac{1}{\sigma_{i}^{\alpha}}\left(\int_{\mathbb{S}^{d}}\left|\left\langle\mathbf{b}_{\mathbf{i}}, \mathbf{s}\right\rangle\right|^{[\alpha]} \Gamma(d \mathbf{s})\right)^{1 / \alpha},
\end{aligned}
$$


where $\Gamma(\cdot)$ is spectral measure and $|x|^{[r]}=\operatorname{sgn}(x)|x|^{r}$; see Samorodnitsky and Taqqu [2]. It follows, from Kuruoglu [17], that if $X \sim S(\alpha, \beta, \sigma, 0)$, then

$$
\begin{aligned}
& e(\alpha, \beta, \sigma)=E\left(\log ^{2}|X|\right) \\
& =\frac{7 \pi^{2}-6 \theta^{2}+6[\log (\sigma / \cos (\theta))+\gamma(1-\alpha)]^{2}-\pi^{2} \alpha^{2}}{6 \alpha^{2}},
\end{aligned}
$$

where $\theta=\arctan (\beta \tan (\pi \alpha / 2))$. Also,

$$
\begin{aligned}
& e\left(\alpha, \beta_{i}, \sigma_{i}\right)=E\left(\log ^{2}|Z|\right) \\
& =\frac{7 \pi^{2}-6 \theta_{i}^{2}+6\left[\log \left(\sigma_{i} / \cos \left(\theta_{i}\right)\right)+\gamma(1-\alpha)\right]^{2}-\pi^{2} \alpha^{2}}{6 \alpha^{2}},
\end{aligned}
$$

where $Z=-\sum_{j=1}^{i} X_{j}+X_{i+1}+\cdots+X_{d}$, for $i=0, \ldots, d$ and $\theta_{i}=\arctan \left(\beta_{i} \tan (\pi \alpha / 2)\right)$. Parameters $\sigma_{i}$ and $\beta_{i}$ are defined in (A.5). Finally,

$$
E\left(\log ^{2}\|\mathbf{X}\|\right) \leq e(\alpha, \beta, \sigma)+\sum_{i=0}^{d}\left(\begin{array}{l}
d \\
i
\end{array}\right) e\left(\alpha, \beta_{i}, \sigma_{i}\right) .
$$

The proof is complete since all terms on the right-hand side of (A.8) are finite.

Proof of Theorem 4. We rewrite Definition 1 as

$$
A \mathbf{X}_{1}+B \mathbf{X}_{2} \stackrel{d}{=} C \mathbf{X}_{1}+\mathbf{D}
$$

Setting $A=1, B=1$, and $\mathbf{D}=\mathbf{0}$ in (A.9), it yields

$$
\mathbf{X}_{1}+\mathbf{X}_{2} \stackrel{d}{=} 2^{1 / \alpha} \mathbf{X}_{1}
$$

By applying log-transformation, after taking the Euclidean norm, to both sides of (A.10), we have

$$
\frac{1}{\alpha}=\frac{\log \left\|\mathbf{X}_{1}+\mathbf{X}_{2}\right\|-\log \left\|\mathbf{X}_{1}\right\|}{\log 2} .
$$

The right-hand side of (A.11) can be used to define a symmetric kernel of the form

$$
\begin{aligned}
H\left(\mathbf{X}_{1}, \mathbf{X}_{2}\right)= & \frac{\log \left\|\mathbf{X}_{1}+\mathbf{X}_{2}\right\|}{\log 2} \\
& -\frac{\log \left\|\mathbf{X}_{1}\right\|+\log \left\|\mathbf{X}_{2}\right\|}{2 \log 2} .
\end{aligned}
$$

To guarantee the asymptotic normality of the introduced $U$ statistics for $1 / \alpha$ with kernel (A.12), we need to check that $E\left(H\left(\mathbf{X}_{1}, \mathbf{X}_{2}\right)\right)^{2}<\infty$. It suffices to show that $\operatorname{Var} H\left(\mathbf{X}_{1}, \mathbf{X}_{2}\right)<$ $\infty$. For this, the result of Lemma 3 shows that $\operatorname{Var} \log \left\|\mathbf{X}_{1}\right\|$ is finite. On the other hand, from (A.10) it turns out that

$$
\text { Var } \log \left\|\mathbf{X}_{1}+\mathbf{X}_{2}\right\|=\text { Var } \log \left\|\mathbf{X}_{1}\right\|=\operatorname{Var} \log \left\|\mathbf{X}_{2}\right\| \text {. }
$$

We use property (A.13) to calculate variance of the right-hand side of (A.12) as

$$
\begin{aligned}
\operatorname{Var} H\left(\mathbf{X}_{1}, \mathbf{X}_{2}\right)= & \frac{\operatorname{Var} \log \left\|\mathbf{X}_{1}\right\|}{\log ^{2} 2}+\frac{\operatorname{Var} \log \left\|\mathbf{X}_{1}\right\|}{2 \log ^{2} 2} \\
& -\frac{K}{\log ^{2} 2},
\end{aligned}
$$

where

$$
\begin{aligned}
K & =\operatorname{Cov}\left(\log \left\|\mathbf{X}_{1}+\mathbf{X}_{2}\right\|, \log \left\|\mathbf{X}_{1}\right\|+\log \left\|\mathbf{X}_{2}\right\|\right) \\
& \leq 2 \sqrt{\operatorname{Var} \log \left\|\mathbf{X}_{1}+\mathbf{X}_{2}\right\|} \sqrt{\operatorname{Var} \log \left\|\mathbf{X}_{1}\right\| .}
\end{aligned}
$$

Applying property (A.13) again on the right-hand side of (A.15), we have

$$
\operatorname{Var} H\left(\mathbf{X}_{1}, \mathbf{X}_{2}\right) \leq \frac{7 \operatorname{Var} \log \left\|\mathbf{X}_{1}\right\|}{2 \log ^{2} 2},
$$

where we used the result of Lemma 3 to get the right-hand side of (A.16). This means that

$$
\begin{aligned}
\lambda & =\operatorname{Var}\left(E\left(H\left(\mathbf{X}_{1}, \mathbf{X}_{2}\right) \mid \mathbf{X}_{1}\right)\right) \leq \operatorname{Var} H\left(\mathbf{X}_{1}, \mathbf{X}_{2}\right) \\
& <\infty .
\end{aligned}
$$

Therefore, $E\left(H\left(\mathbf{X}_{1}, \mathbf{X}_{2}\right)\right)^{2}<\infty$. Now, we define $U$-statistic for $1 / \alpha$ with kernel given in (A.12) as

$$
U_{n}=\left(\begin{array}{l}
n \\
2
\end{array}\right)^{-1} \sum_{1 \leq i<j \leq n} H\left(\mathbf{x}_{i}, \mathbf{x}_{j}\right) .
$$

By definition, given $U$-statistic in (A.18) is unbiased estimator for $1 / \alpha$.

\section{Conflicts of Interest}

The authors declare that they have no conflicts of interest.

\section{References}

[1] P. Lévy, "Théorie des erreurs. La loi de Gauss et les lois exceptionnelles," Bulletin De La Société Mathématique De France, vol. 52, pp. 49-85, 1924.

[2] G. Samorodnitsky and M. S. Taqqu, Stable Non-Gaussian Random Processes: Stochastic Models and Infinite Variance, Stochastic Modeling, Chapman \& Hall, New York, NY, USA, 1994.

[3] J. P. Nolan, "Maximum likelihood estimation of stable parameters," in Lévy Processes: Theory and Applications, O. E. BarndorffNielsen, T. Mikosch, and I. Resnick, Eds., pp. 379-400, Birkhöuser, Boston, Mass, USA, 2001.

[4] J. P. Nolan, "Numerical calculation of stable densities and distribution functions," Communications in Statistics. Stochastic Models, vol. 13, no. 4, pp. 759-774, 1997.

[5] J. H. McCulloch, "Simple consistent estimators of stable distribution parameters," Communications in Statistics-Simulation and Computation, vol. 15, no. 4, pp. 1109-1136, 1986.

[6] S. M. Kogon and D. B. Williams, "Characteristic function based estimation of stable parameters," in A Practical Guide to Heavy Tailed Data, R. Adler, R. Feldman, and M. Taqqu, Eds., pp. 311338, Birkhäuser, Boston, Mass, USA, 1998.

[7] Z. Fan, "Parameter estimation of stable distributions," Communications in Statistics. Theory and Methods, vol. 35, no. 1-3, pp. 245-255, 2006.

[8] J. P. Nolan, A. K. Panorska, and J. H. McCulloch, "Estimation of stable spectral measures," Mathematical and Computer Modelling, vol. 34, no. 9-11, pp. 1113-1122, 2001. 
[9] M. Pivato and L. Seco, "Estimating the spectral measure of a multivariate stable distribution via spherical harmonic analysis," Journal of Multivariate Analysis, vol. 87, no. 2, pp. 219240, 2003.

[10] H. Ogata, "Estimation for multivariate stable distributions with generalized empirical likelihood," Journal of Econometrics, vol. 172, no. 2, pp. 248-254, 2013.

[11] M. Mohammadi, A. Mohammadpour, and H. Ogata, "On estimating the tail index and the spectral measure of multivariate $\alpha \alpha$-stable distributions," Metrika, vol. 78, no. 5, pp. 549-561, 2015.

[12] T. Byczkowski, J. P. Nolan, and B. Rajput, "Approximation of multidimensional stable densities," Journal of Multivariate Analysis, vol. 46, no. 1, pp. 13-31, 1993.

[13] J. H. McCulloch, "Estimation of the bivariate stable spectral representation by the projection method," Computational Economics, vol. 16, no. 1-2, pp. 47-62, 2000.

[14] R. Modarres and J. P. Nolan, "A method for simulating stable random vectors," Computational Statistics, vol. 9, no. 1, pp. 1119, 1994.

[15] Robust Analysis Inc, User Manual for STABLE 5.0, Software and User Manual, 2010, http://www.robustanalysis.com.

[16] J. P. Nolan, "Multivariate elliptically contoured stable distributions: theory and estimation," Computational Statistics, vol. 28, no. 5, pp. 2067-2089, 2013.

[17] E. E. Kuruoglu, "Density parameter estimation of skewed /spl alpha/-stable distributions," IEEE Transactions on Signal Processing, vol. 49, no. 10, pp. 2192-2201, 2001. 


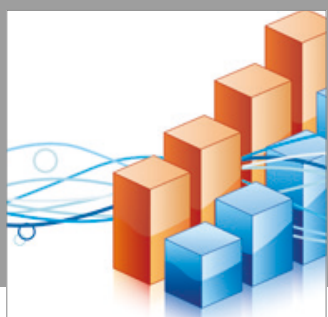

Advances in

Operations Research

vatersals

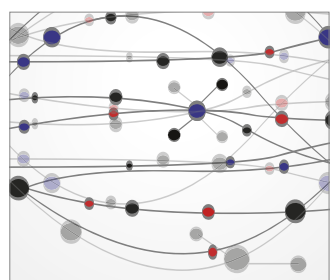

\section{The Scientific} World Journal
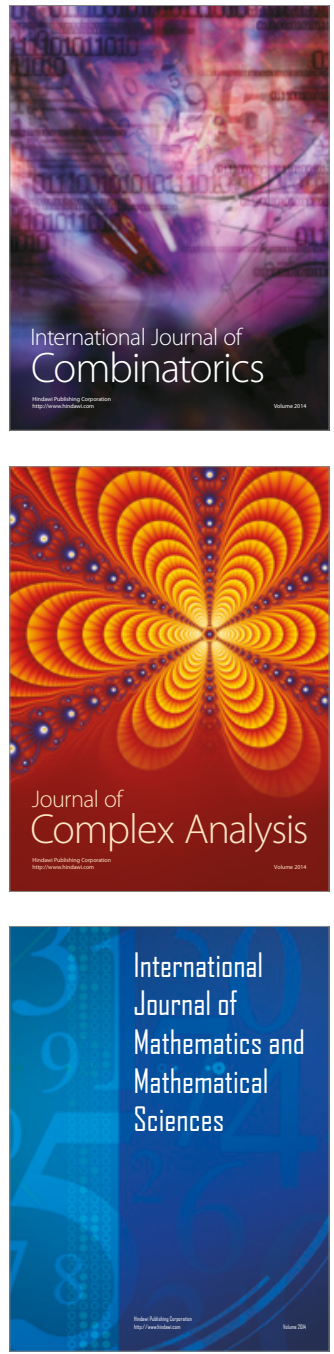
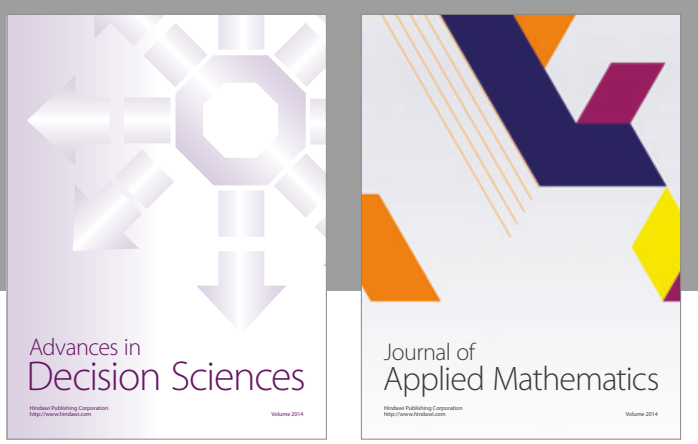

Algebra

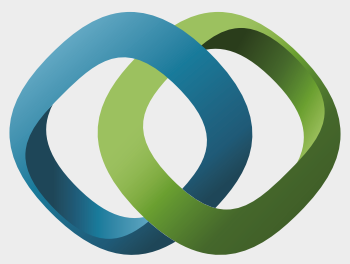

\section{Hindawi}

Submit your manuscripts at

https://www.hindawi.com
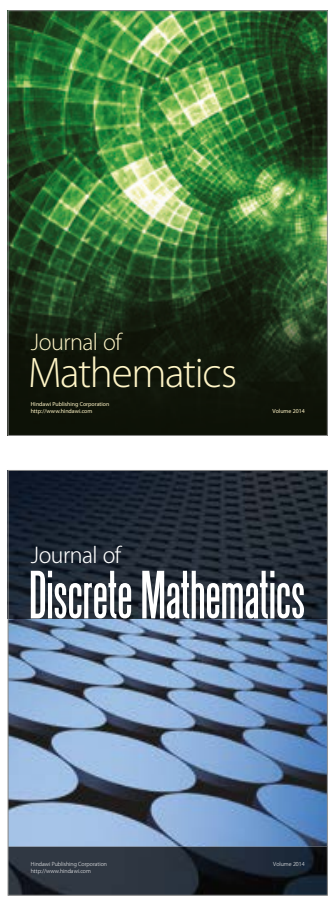

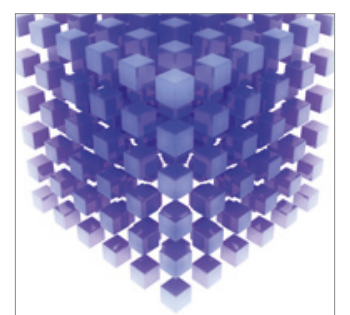

Mathematical Problems in Engineering
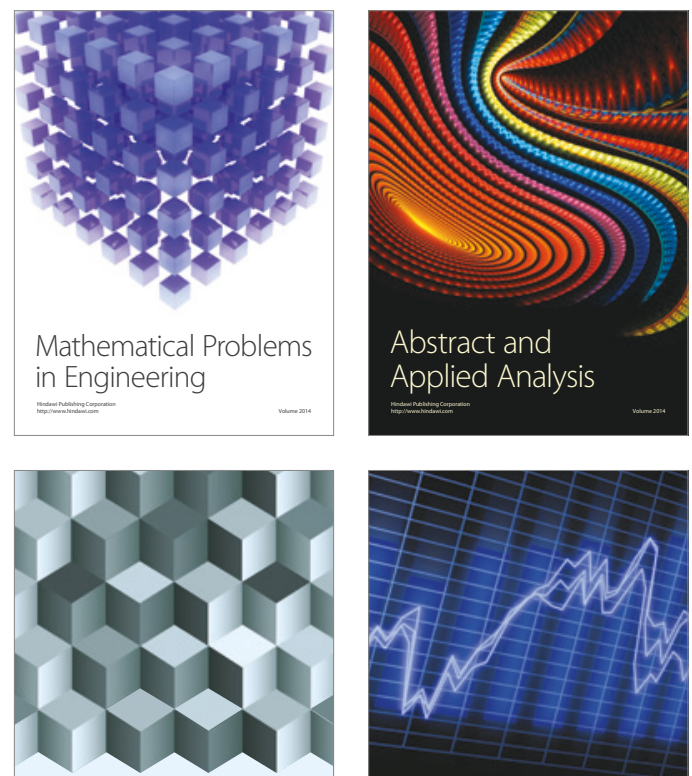

Journal of

Function Spaces

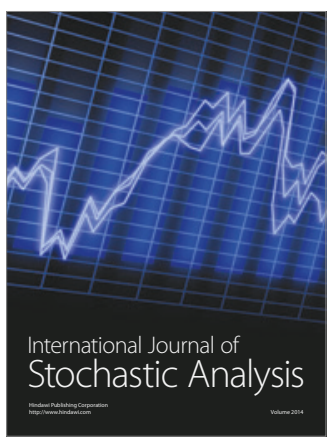

Probability and Statistics
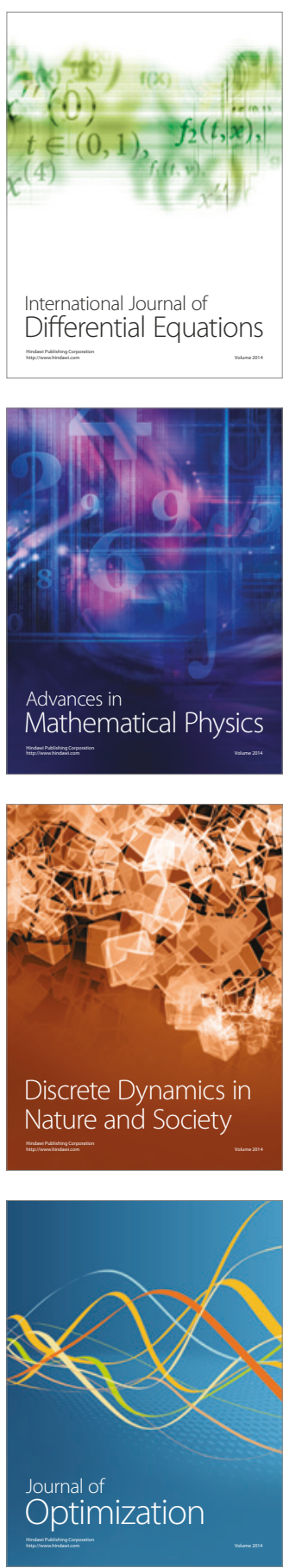